University of Nebraska - Lincoln

DigitalCommons@University of Nebraska - Lincoln

US Department of Energy Publications

U.S. Department of Energy

2003

Deformation of a Peridynamic Bar

S. A. Silling

Sandia National Laboratories

M. Zimmermann

Massachusetts Institute of Technology

R. Abeyaratne

Massachusetts Institute of Technology

Follow this and additional works at: https://digitalcommons.unl.edu/usdoepub

Part of the Bioresource and Agricultural Engineering Commons

Silling, S. A.; Zimmermann, M.; and Abeyaratne, R., "Deformation of a Peridynamic Bar" (2003). US Department of Energy Publications. 93.

https://digitalcommons.unl.edu/usdoepub/93

This Article is brought to you for free and open access by the U.S. Department of Energy at DigitalCommons@University of Nebraska - Lincoln. It has been accepted for inclusion in US Department of Energy Publications by an authorized administrator of DigitalCommons@University of Nebraska - Lincoln. 


\title{
Deformation of a Peridynamic Bar
}

\author{
S.A. SILLING ${ }^{1}$, M. ZIMMERMANN ${ }^{2}$ and R. ABEYARATNE ${ }^{2}$ \\ ${ }^{1}$ Computational Physics Department, MS-0820, Sandia National Laboratories, Albuquerque, \\ NM 87185-0820, U.S.A. E-mail: sasilli@sandia.gov \\ ${ }^{2}$ Department of Mechanical Engineering, Room 3-173, Massachusetts Institute of Technology, \\ Cambridge, MA 02139, U.S.A.
}

Received 18 March 2003

\begin{abstract}
The deformation of an infinite bar subjected to a self-equilibrated load distribution is investigated using the peridynamic formulation of elasticity theory. The peridynamic theory differs from the classical theory and other nonlocal theories in that it does not involve spatial derivatives of the displacement field. The bar problem is formulated as a linear Fredholm integral equation and solved using Fourier transform methods. The solution is shown to exhibit, in general, features that are not found in the classical result. Among these are decaying oscillations in the displacement field and progressively weakening discontinuities that propagate outside of the loading region. These features, when present, are guaranteed to decay provided that the wave speeds are real. This leads to a onedimensional version of St. Venant's principle for peridynamic materials that ensures the increasing smoothness of the displacement field remotely from the loading region. The peridynamic result converges to the classical result in the limit of short-range forces. An example gives the solution to the concentrated load problem, and hence provides the Green's function for general loading problems.
\end{abstract}

Mathematics Subject Classifications (2000): 74B20, 82B21, 74A99, 45A05, 45B05.

Key words: linear integral equations, Fredholm integral equations, peridynamic, Fourier transform, discontinuity, Green's function, point load, elastic bar.

\section{Peridynamic Theory}

Discontinuities of various sorts arise naturally in the modeling of many phenomena of physical interest, including cracks. Since the classical theory of elasticity involves spatial derivatives of the displacement field that may not exist on these singularities, an alternative formulation of elasticity theory, one that avoids the use of any spatial derivatives, has been proposed by Silling [1]. This formulation, called the peridynamic theory, uses integral equations to compute the force on a material particle, and therefore, in general, it can account for both short-range and long-range forces.

The peridynamic approach may be thought of as a continuum version of molecular dynamics, because material particles interact with each other directly through finite distances. In the three-dimensional peridynamic theory, the equation of motion is given by

$$
\rho \ddot{\mathbf{u}}(\mathbf{x}, t)=\int_{\mathcal{R}} \mathbf{f}\left(\mathbf{x}, \mathbf{x}^{\prime}, t\right) \mathrm{d} V^{\prime}+\mathbf{b}(\mathbf{x}, t)
$$


where $\mathcal{R}$ is a region, $\mathbf{x}$ is the location of a particle in the reference configuration, $\mathbf{u}$ is the displacement field, $\rho$ is the mass density, $\mathbf{b}$ is a prescribed body force field, and $\mathbf{f}$ is the force density on particle $\mathbf{x}$ due to particle $\mathbf{x}^{\prime}$ at time $t$. To arrive at a peridynamic one-dimensional theory for an infinitely long bar of constant crosssection with area $A$, assume that the bar is parallel to the $x_{1}$ axis. Then integrate (1) over the cross-section $\mathcal{A}$ located at any $x_{1}$ and divide through by $A$ to obtain

$$
\rho \ddot{u}(x, t)=\int_{-\infty}^{\infty} f\left(x, x^{\prime}, t\right) \mathrm{d} x^{\prime}+b(x, t),
$$

where $x \equiv x_{1}$,

$$
u\left(x_{1}, t\right)=\frac{1}{A} \int_{\mathscr{A}} u_{1}(\mathbf{x}, t) \mathrm{d} A, \quad b\left(x_{1}, t\right)=\frac{1}{A} \int_{\mathscr{A}} b_{1}(\mathbf{x}, t) \mathrm{d} A
$$

and

$$
f\left(x_{1}, x_{1}^{\prime}, t\right)=\frac{1}{A} \int_{\mathcal{A}} \int_{\mathcal{A}^{\prime}} f_{1}\left(\mathbf{x}, \mathbf{x}^{\prime}, t\right) \mathrm{d} A^{\prime} \mathrm{d} A
$$

in which $\mathrm{d} V^{\prime}=\mathrm{d} A^{\prime} \mathrm{d} x^{\prime}$ has been used. Note that the one-dimensional force density $f$ is not equal to the three-dimensional force density component $f_{1}$; in fact, these two quantities do not have the same units.

Because the peridynamic theory is nonlocal, the displacement components $u_{2}$ and $u_{3}$ are not necessarily linear functions of $x_{2}$ and $x_{3}$. This makes it much less straightforward to derive the constitutive model for a bar from a three-dimensional constitutive model in the peridynamic theory than in the classical theory, e.g., Atkin and Fox [2]. Instead, we formulate the constitutive law for a peridynamic bar directly in the one-dimensional setting, omitting the calculations that would be required by (4).

The constitutive response of the bar is characterized by a function $\hat{f}$ which relates the force between particles to the kinematics, which for a homogeneous objective microelastic material has the form

$$
f\left(x, x^{\prime}, t\right)=\hat{f}\left(u\left(x^{\prime}, t\right)-u(x, t), x^{\prime}-x\right) .
$$

For consistency with Newton's third law, $\hat{f}$ must have the symmetry property

$$
\hat{f}(\eta, \xi)=-\hat{f}(-\eta,-\xi) \quad \forall \eta, \xi
$$

One way in which to see the connection between the peridynamic theory and the classical theory is to consider, for example, the following special form of the constitutive function $\hat{f}$ :

$$
\hat{f}(\eta, \xi)=-2 \hat{\sigma}\left(\frac{\eta}{\xi}\right) d_{\ell}^{\prime}(\xi), \quad \xi \neq 0 .
$$

Here $\hat{\sigma}(\cdot)$ is a continuously differentiable function on $(-\infty, \infty)$. The sequence of functions $d_{\ell}(\xi)$ parameterized by $\ell$ is a generalized function in the sense of, 
e.g., [3], which implies that, as $\ell \rightarrow 0$, this sequence approaches a delta function; $d_{\ell}^{\prime}(\xi)$ is its derivative with respect to $\xi$. In view of $(6), d_{\ell}(\cdot)$ must be an even function. By substituting (7) into (5), and the result into the integral in (2), changing the dummy variable from $x^{\prime}$ to $\xi=x^{\prime}-x$, integrating by parts, and using the delta function-like properties of $d_{\ell}$ as $\ell \rightarrow 0$, one can readily verify that in the limit $\ell \rightarrow 0$, the integral in (2) yields

$$
\begin{aligned}
& \lim _{\ell \rightarrow 0} \int_{-\infty}^{\infty} f\left(x^{\prime}, x, t\right) \mathrm{d} x^{\prime} \\
& \quad=-2 \lim _{\ell \rightarrow 0} \int_{-\infty}^{\infty} \hat{\sigma}\left(\frac{u(x+\xi, t)-u(x, t)}{\xi}\right) \frac{\mathrm{d}}{\mathrm{d} \xi} d_{\ell}(\xi) \mathrm{d} \xi \\
& \quad=2 \lim _{\xi \rightarrow 0} \frac{\mathrm{d}}{\mathrm{d} \xi} \hat{\sigma}\left(\frac{u(x+\xi, t)-u(x, t)}{\xi}\right)=\hat{\sigma}^{\prime}\left(u_{x}\right) u_{x x} .
\end{aligned}
$$

Therefore the peridynamic equation of motion (2) reduces to the classical equation of motion in the limit $\ell \rightarrow 0$ :

$$
\rho \ddot{u}=\frac{\partial \sigma}{\partial x}+b \quad \text { with } \sigma=\hat{\sigma}(\varepsilon), \varepsilon=\frac{\partial u}{\partial x} .
$$

The parameter $\ell$ in the constitutive description (7) therefore plays the role of a length-scale parameter and one recovers the classical theory of nonlinear elasticity in the limit of vanishing length scale. This is the limit of short range forces.

In this paper we shall be working with linear peridynamic materials where

$$
\hat{f}(\eta, \xi)=C(\xi) \eta
$$

$C$ is referred to as the material's micromodulus function. For such a material, the equation of motion specializes to

$$
\rho \ddot{u}(x, t)=\int_{-\infty}^{\infty} C\left(x^{\prime}-x\right)\left(u\left(x^{\prime}, t\right)-u(x, t)\right) \mathrm{d} x^{\prime}+b(x, t) .
$$

We require two characteristics of the micromodulus function $C$ : First, in view of (6), $C$ must be an even function:

$$
C(\xi)=C(-\xi) \text { for }-\infty<\xi<\infty .
$$

Second, if the material is to sustain real propagating sinusoidal waves of arbitrary wave number, the micromodulus $C$ must be suitably restricted. Substituting $u(x, t)=\exp \mathrm{i}(\kappa x-\omega t)$ into the equation of motion (11) leads to the following dispersion relation between the angular frequency $\omega$ and the wave number $\kappa$ :

$$
\rho \omega^{2}=M(\kappa) .
$$

Here

$$
M(\kappa)=\int_{-\infty}^{\infty}(1-\cos \kappa \xi) C(\xi) \mathrm{d} \xi
$$


and it is the one-dimensional specialization of the acoustic tensor discussed in [1]. It relates the constitutive properties contained in $C$ to the wave speeds $c=c(\kappa)=$ $\omega / \kappa$ of the material. Note that in the linear peridynamic theory, unlike the classical theory of linear elasticity, wave speeds are in general dependent on the wave number. We require that the wave speeds corresponding to every wave number be real, which means that the micromodulus $C$ must be such that

$$
M(\kappa)>0 \quad \text { whenever } \kappa \neq 0 .
$$

This requirement is analogous to strong ellipticity in the classical theory. Note that $C$ is not required to be positive everywhere since this is sufficient, but not necessary, for (15) to hold; on the other hand, one can show that $E>0$ in (20) below is necessary but not sufficient. (See [1] for a detailed discussion of waves in linear peridynamic materials.) Note trivially from (14) that $M(0)=0$, and so, when (15) holds, $M$ has no real zeros other than $\kappa=0$.

Next, suppose that particles $x$ and $x^{\prime}$ which are further away than some critical distance do not interact. This means that $C$ possesses a horizon $h$ such that it vanishes identically outside the horizon:

$$
C(\xi)=0 \text { for }|\xi|>h \text {. }
$$

It is not necessary that the micromodulus function have a finite horizon, though one would expect on physical grounds that $C(\xi) \rightarrow 0$ as $\xi \rightarrow \pm \infty$. With regard to the smoothness of the micromodulus, suppose that $C \in \mathcal{C}_{\mathrm{pw}}^{N}[-h, h]$, so that $C$ has continuous derivatives of order $0,1, \ldots, N-1$ on $[-h, h]$, but its $N$ th derivative involves discontinuities and is piecewise continuous on this domain.

Finally, although the concept of a stress tensor plays no fundamental role in the peridynamic theory, it is helpful to introduce such a notion in order to make the connection between the peridynamic and classical theories. To do this in one dimension, one can adapt Cauchy's notion of stress in a crystal and define the "stress" $\sigma(x)$ at the particle $x$ to be the total force that all material particles to its right exert on all material particles to its left. Thus by considering the force exerted by the particle located at $x+r$ on the particle located at $x-s$, and summing over all $r>0, s>0$, the stress at $x$ can be defined as

$$
\sigma(x)=\int_{0}^{\infty} \int_{0}^{\infty} \hat{f}(u(x+r)-u(x-s), r+s) \mathrm{d} s \mathrm{~d} r .
$$

In the special case of a homogeneous deformation of the form $u(x)=\varepsilon x$ for some constant $\varepsilon$, the expression (17) simplifies to the (in general nonlinear) "stress-strain relation"

$$
\sigma=\int_{0}^{\infty} \xi \hat{f}(\varepsilon \xi, \xi) \mathrm{d} \xi
$$

note that this is independent of $x$. If the peridynamic material is linear, this further specializes to

$$
\sigma=\varepsilon \int_{0}^{\infty} \xi^{2} C(\xi) \mathrm{d} \xi
$$


and therefore the Young's modulus $E$ in the classical theory of elasticity is related to the micromodulus $C$ in the peridynamic theory by

$$
E=\int_{0}^{\infty} \xi^{2} C(\xi) \mathrm{d} \xi
$$

The relations (18)-(20) are physically meaningful only in the context of homogeneous deformations. Note also that the value of $E$ found in (20) depends not only on the material, but also on the geometry of the cross-section because of (4).

\section{Self-equilibrated Load Problem}

In the classical theory of elasticity, the equilibrium of a bar under a self-equilibrated load is essentially a trivial problem. However the solution according to the peridynamic theory exhibits some interesting phenomena because, here, particles interact with each other through a finite distance. The form of the peridynamic solution away from the loading region is of particular interest. We will see in this section that the spatial variation of the displacement field is a decaying oscillation; and in Section 6 we see further that discontinuities propagate away from the loading region in a progressively weakening manner.

Let a bar occupying $-\infty<x<\infty$ be subjected to a time-independent body force density function $b$ that is self-equilibrated:

$$
\int_{-\infty}^{\infty} b(x) \mathrm{d} x=0 .
$$

Moreover, suppose that the body force vanishes outside some loading region $-a \leqslant$ $x \leqslant a$ :

$$
b(x)=0 \text { for }|x|>a .
$$

Assume that the bar is homogeneous and composed of a linear peridynamic material with micromodulus $C$. The displacement field $u$ is then governed by the linear Fredholm integral equation

$$
\int_{-\infty}^{\infty} C(\xi)(u(x-\xi)-u(x)) \mathrm{d} \xi+b(x)=0, \quad-\infty<x<\infty .
$$

Given $b$ and $C$ subject to the restrictions (21), (22) and (12), (15), (16) we are to determine $u$. Note that, if $C$ possesses a horizon $h$, then the limits of integration in (23) can be written as $\pm h$.

If $u(x)$ is a solution of (23), one can readily verify that $u(x)+K_{1}+K_{2} x$ is also a solution for arbitrary constants $K_{1}$ and $K_{2}$. Thus one does not expect the solution of (23) to be unique. However, there is a close connection between positive wave speeds and positive definiteness of the energy density in the linear peridynamic theory [1] and so it is straightforward to demonstrate uniqueness to within an arbitrary linear function. 
For any function $p$ on $(-\infty, \infty)$, let $\bar{p}$ denote its Fourier transform,

$$
\bar{p}(\kappa)=\int_{-\infty}^{\infty} \mathrm{e}^{-\mathrm{i} \kappa x} p(x) \mathrm{d} x, \quad-\infty<\kappa<\infty,
$$

provided this integral exists. The inverse transform is provided by

$$
p(x)=\frac{1}{2 \pi} \int_{-\infty}^{\infty} \mathrm{e}^{\mathrm{i} \kappa x} \bar{p}(\kappa) \mathrm{d} \kappa, \quad-\infty<x<\infty .
$$

We use the broad interpretation of a Fourier transform where it can be applied, in particular, to delta functions and to functions which do not vanish at $\pm \infty$, e.g., $[3,4]$.

Taking the Fourier transform of the governing equation (23) and using the convolution theorem leads to the following expression for the transform of the displacement field:

$$
\bar{u}(\kappa)=\frac{\bar{b}(\kappa)}{M(\kappa)}, \quad \text { where } M(\kappa)=\bar{C}(0)-\bar{C}(\kappa)
$$

is the function encountered previously in (14). Applying the inversion formula (25) to (26) results in a formal solution to the self-equilibrated load problem:

$$
u(x)=\frac{1}{2 \pi} \int_{-\infty}^{\infty} \frac{\bar{b}(\kappa) \mathrm{e}^{\mathrm{i} \kappa x}}{M(\kappa)} \mathrm{d} \kappa, \quad-\infty<x<\infty .
$$

Reversing the above steps confirms that this formal solution indeed solves the original problem provided the functions involved are such that the forward and inverse Fourier transforms can be applied to them.

\section{Remote Behavior}

We now examine the displacement field (27) at points far from the loading region $-a \leqslant x \leqslant a$. With a view toward using the method of contour integration, the independent variable in the Fourier transform will now be allowed to take on complex values. The notation $\bar{p}(z)$ instead of $\bar{p}(\kappa)$ will be used when the Fourier transform is being considered as a function on the complex plane with $z=\kappa+\mathrm{i} \nu$ where $\kappa$ and $v$ are real numbers.

Assume that $\bar{b}$ exists and is bounded. Then $\bar{b}(z)$ is entire and therefore the only poles of the integrand in (27) are the zeros of the function $M(z)$. As noted previously, $z=0$ is the unique real zero of $M$. Also, $M$ generally has complex zeros as well, depending on the material. If these complex zeros are present, because of the symmetry condition (12), they occur in sets of four located symmetrically in the four quadrants of the $z$-plane as shown in Figure 1 . Let $z_{j}, j=1,2, \ldots, Q$, denote the zeros of $M$ in the first quadrant and let $\kappa_{j}$ and $v_{j}$ be the associated real and imaginary parts:

$$
z_{j}=\kappa_{j}+\mathrm{i} v_{j}, \quad \kappa_{j} \geqslant 0, v_{j}>0, j=1,2, \ldots, Q
$$




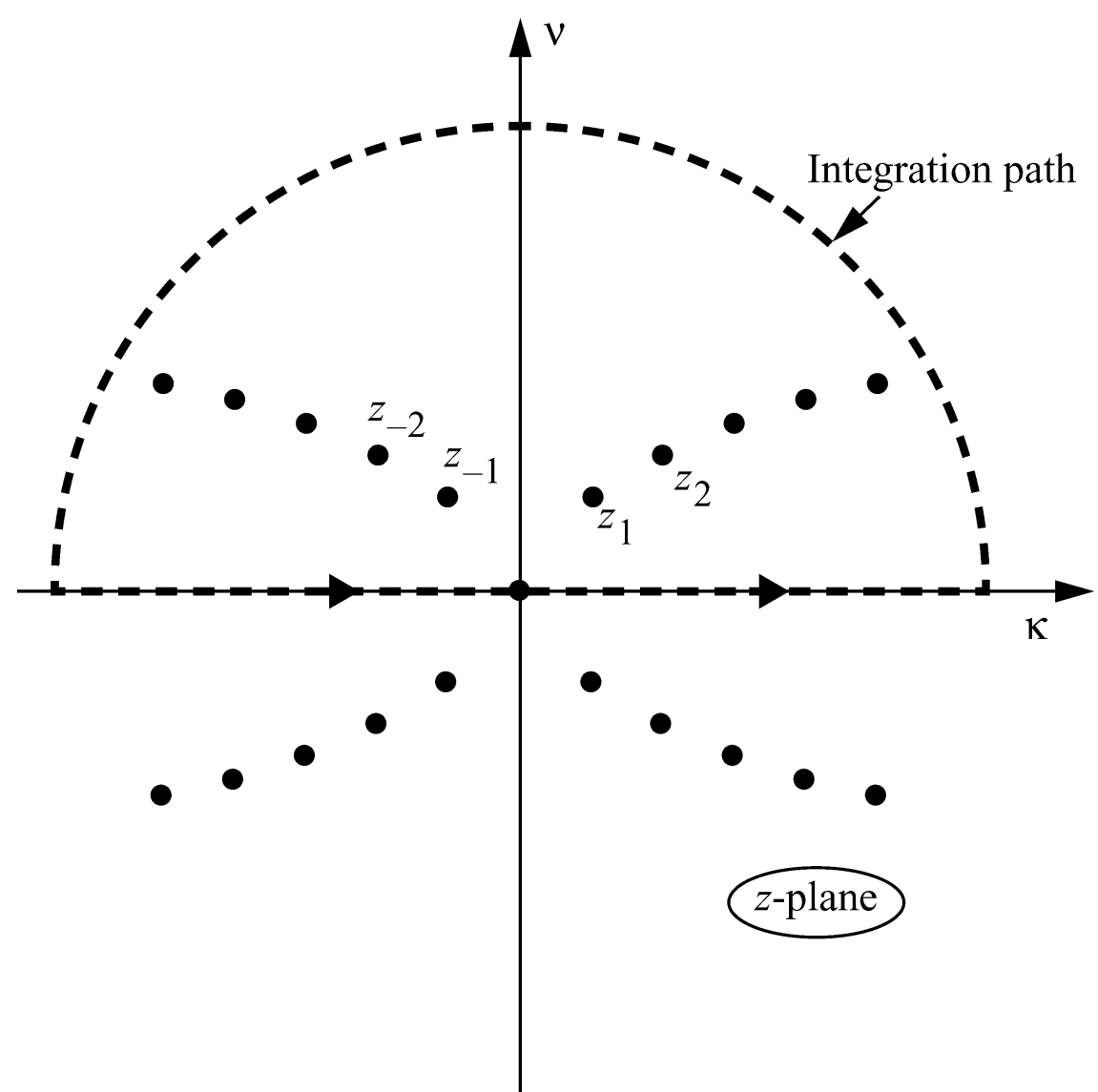

Figure 1. Roots of $M(z)$ in the complex plane. The semicircular contour used for inverting the Fourier transform is also shown.

the number of complex zeros, $4 Q$, may be finite or infinite. The strict inequality on $v_{j}$ follows from the fact that $z_{j}$ cannot be real. If we denote the zeros in the second quadrant by $z_{-j}, j=1,2, \ldots, Q$, then necessarily

$$
z_{-j}=-\kappa_{j}+\mathrm{i} v_{j}, \quad j=1,2, \ldots, Q .
$$

Thus the zeros in the upper half plane are $z_{j}, j= \pm 1, \pm 2, \ldots, \pm Q$, and those in the lower half-plane are $-z_{j}, j= \pm 1, \pm 2, \ldots, \pm Q$.

Since $\bar{b}$ is bounded, and because $a>0$, the term $\left|\mathrm{e}^{\mathrm{i} z x} \bar{b}(z)\right|$ decreases exponentially in the upper half-plane for large $|z|$ when $x>a$; likewise for $x<-a$, it decreases exponentially in the lower half-plane. In order to evaluate the integral in (27) by the method of residues one must therefore complete the contour of integration with a large semi-circle in the upper half-plane for $x>a$, and by a large semi-circle in the lower half-plane for $x<-a$. Moreover, the path along the real axis has to avoid the pole at the origin by appropriately going below and above it in these respective cases, and taking one-half the residue at the origin in each 
case. In order to calculate the residue at the origin we first note from (14) and (20) that

$$
M(0)=0, \quad M^{\prime}(0)=0, \quad M^{\prime \prime}(0)=2 E,
$$

and from (21) and (24) that

$$
\bar{b}(0)=\int_{-\infty}^{\infty} b(x) \mathrm{d} x=0, \quad \bar{b}^{\prime}(0)=-\mathrm{i} \int_{-\infty}^{\infty} \xi b(\xi) \mathrm{d} \xi .
$$

Therefore $z=0$ is a simple pole and the corresponding residue of (27) is

$$
R_{0}=\operatorname{Res}_{z=0}\left\{\frac{\bar{b}(z) \mathrm{e}^{\mathrm{i} z x}}{2 \pi M(z)}\right\}=\frac{\bar{b}^{\prime}(0)}{\pi M^{\prime \prime}(0)}=\frac{-\mathrm{i}}{2 \pi E} \int_{-\infty}^{\infty} \xi b(\xi) \mathrm{d} \xi .
$$

Similarly let $R_{j}^{ \pm}$denote the residue at $\pm z_{j}$ :

$$
R_{j}^{ \pm}(x)=\operatorname{Res}_{z= \pm z_{j}}\left\{\frac{\bar{b}(z) \mathrm{e}^{\mathrm{i} z x}}{2 \pi M(z)}\right\}, \quad j= \pm 1, \pm 2, \ldots, \pm Q .
$$

By using these results and applying the residue theorem, we find that the displacement field (27) outside of the loading region can be written explicitly as

$$
u(x)= \begin{cases}u_{\infty}+2 \pi \mathrm{i} \sum_{j=-Q, j \neq 0}^{Q} R_{j}^{+}(x), & x>a, \\ -u_{\infty}-2 \pi \mathrm{i} \sum_{j=-Q, j \neq 0}^{Q} R_{j}^{-}(x), & x<-a,\end{cases}
$$

where we have set

$$
u_{\infty}=\pi \mathrm{i} R_{0}=\frac{1}{2 E} \int_{-\infty}^{\infty} \xi b(\xi) \mathrm{d} \xi
$$

Since $v_{j}>0$ for each integer $j>0$, it follows from (28), (29) and (33) that $R_{j}^{ \pm}(x) \rightarrow 0$ as $x \rightarrow \pm \infty$. Therefore $u(x) \rightarrow \pm u_{\infty}$ as $x \rightarrow \pm \infty$. Therefore the relative displacement between the two ends of the bar is $2 u_{\infty}$. Integration of the right side of (35) by parts shows, perhaps surprisingly, that this value agrees with the corresponding result in the classical theory for a bar with zero force applied at the ends.

Yet, the displacement field (34) has an interesting structure which is different from that of the classical theory. To see this, note from (28), (29), (33) and (35) that $(34)_{1}$ can be written in the following form:

$$
u(x)=u_{\infty}+\sum_{j=1}^{Q} \mathrm{e}^{-v_{j} x}\left(A_{j} \cos \kappa_{j} x+B_{j} \sin \kappa_{j} x\right), \quad x>a,
$$


for some set of real constants $A_{j}$ and $B_{j}$. Since by (28) all the $v_{j}$ 's are positive, the trigonometric terms in (36) represent oscillatory structures that decay as one moves away from the region in which the load is applied.

Recall that $M(z)$ always has the origin as its unique real zero, but it may fail to have complex zeros. This case corresponds to $Q=0$ in the above discussion, so that $u(x)=u_{\infty}$ for $x>a$ according to (34). Therefore, no oscillations are present in such a material outside the loading region. (The microelastic material with $C(x)=\mathrm{e}^{-\lambda|x|}$, where $\lambda$ is a positive constant, is an example of a material with this property. We have studied the self-equilibrated load problem for this material in detail, for both infinite- and finite-length bars, as will be reported separately.)

Recall from (13) that it is the requirement of real wave speeds that implied that $M$ has no real zeros other than 0 . If there had been another real zero, then the corresponding $v$ would vanish, and so the oscillations in (36) would not decay as $x \rightarrow \infty$. So real wave speeds ensure reasonable behavior in this equilibrium problem.

Also, since $z_{j}$ is a zero of the function $M$, and since $M$ as defined in (14) depends on the material but not the loading, it follows that the decay rate depends on the nature of the material but not on the loading distribution. So, even if a loading function $b$ has strong high-frequency components, these components are not propagated very far away from the loading region.

Finally, let $v_{\min }$ be the smallest of the $v_{j}$ 's. Then, for large $x$, the corresponding term is damped out slowest and therefore controls the remote behavior of the solution:

$$
u(x) \sim u_{\infty}+\mathrm{e}^{-v_{\min } x}\left(A_{\min } \cos \kappa_{\min } x+B_{\min } \sin \kappa_{\min } x\right) \quad \text { as } x \rightarrow \infty .
$$

Under reasonable conditions, the higher order terms decay increasingly rapidly.

\section{Example: Double Concentrated Load}

We now turn to a specific case of the preceding problem. Suppose that the micromodulus function $C$ is piecewise constant as follows:

$$
C(\xi)= \begin{cases}\frac{3 E}{\ell^{3}}, & |\xi| \leqslant \ell \\ 0, & |\xi|>\ell\end{cases}
$$

The positive constant $E$ can be shown, using (20), to be the Young's modulus; it is independent of $\ell$ which is both the internal length scale and the horizon in this example. By (24), its Fourier transform $\bar{C}$ is

$$
\bar{C}(\kappa)=\frac{6 E}{\ell^{3}} \frac{\sin \kappa \ell}{\kappa} \quad \text { for } \kappa \neq 0, \quad \bar{C}(0)=\frac{6 E}{\ell^{2}} .
$$

Suppose that the bar is subjected to a pair of self-equilibrated concentrated forces, each of unit magnitude, acting at the points $x= \pm a$. Then

$$
b(x)=\delta(x-a)-\delta(x+a),
$$


where $\delta$ is the delta function. By (24), the Fourier transform of $b$ is

$$
\bar{b}(\kappa)=\mathrm{e}^{-\mathrm{i} a \kappa}-\mathrm{e}^{\mathrm{i} a \kappa}=-2 \mathrm{i} \sin a \kappa .
$$

The displacement field in this bar, according to (27), (39) and (41), is given by

$$
U(x)=\frac{-\mathrm{i} \ell^{3}}{6 \pi E} \int_{-\infty}^{\infty} \mathrm{e}^{\mathrm{i} \kappa x} \frac{\kappa \sin a \kappa}{\kappa \ell-\sin \kappa \ell} \mathrm{d} \kappa ;
$$

because we will make use of this particular displacement field in the next section, we denote it by the symbol $U$ rather than the generic $u$. Contour integration may be used as described in Section 3 to evaluate the integral in (42). Let $z_{j}$ denote the zeros in the upper half-plane of the function $(1-\sin z / z)$. After applying the residue theorem, the displacement field in the bar is found to be

$$
\begin{aligned}
U(x)= & U_{0}(x)+\frac{\mathrm{i} \ell}{6 E} \sum_{j= \pm 1, \pm 2, \ldots} \frac{z_{j}\left(\mathrm{e}^{\mathrm{i} z_{j}|x-a| / \ell}-\mathrm{e}^{\mathrm{i} z_{j}|x+a| / \ell}\right)}{1-\cos z_{j}} \\
& +\frac{1}{P}(\delta(x-a)-\delta(x+a)), \quad-\infty<x<\infty,
\end{aligned}
$$

where

$$
U_{0}(x)= \begin{cases}-\frac{a}{E}, & x<-a, \\ \frac{x}{E}, & |x| \leqslant a, \\ \frac{a}{E}, & x>a .\end{cases}
$$

In constructing (43), the special case $x= \pm a$ must be evaluated directly from (42) rather than by using contour integration because no suitable contour can be found. However, the integral is easily evaluated in this case using the identity $2 \pi \delta(x \pm a)=\int_{-\infty}^{\infty} \mathrm{e}^{\mathrm{i} \kappa(x \pm a)} \mathrm{d} \kappa$. The resulting term in (43), which contains the delta functions, is not particular to this material, but would appear for other microelastic materials as well. This can be deduced from the discussion of discontinuities in Section 6 below (see, for example, equation (60)).

When the length scale $\ell \rightarrow 0$, the terms in the summand decay exponentially and therefore (43) agrees with the classical result $u=U_{0}(x)$ in this limit.

Figure 2 shows plots of the displacement field (43) for the three values $\ell / a=$ 4.0, 2.0 and 0.5 . The solutions were obtained using a relaxation method.

\section{Single concentrated load problem}

We now consider an infinite bar subjected to a single concentrated force at the origin (and suitable forces at infinity to hold it in equilibrium). The solution of this problem can be used as a Green's function when the loading is more general. 


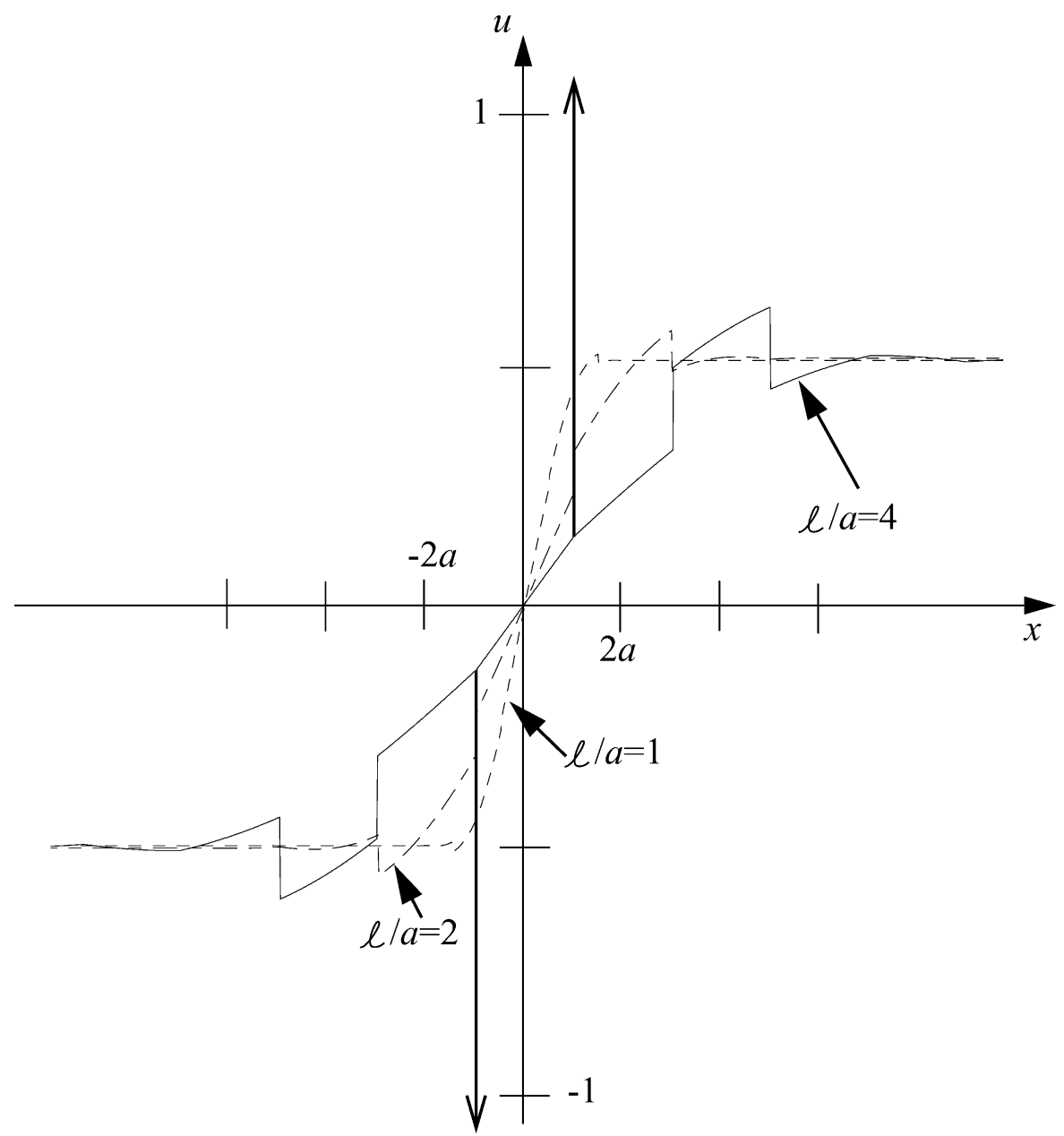

Figure 2. Numerical solution to the double concentrated load problem for materials with three different values of the horizon. The spikes are delta functions at the points where the loads are applied, $\pm a$.

Even though the single load does not correspond to a self-equilibrated loading, a solution to this problem can be found by using the solution from the preceding section together with superposition. From the previous example, the displacement field corresponding to the application of a pair of concentrated forces of magnitude 1 at $x= \pm a$ is $u=U(x)$, where $U$ is given by (43). Therefore the displacement field associated with a pair of forces of magnitude -0.5 applied at $x=0$ and $x=2 a$ is $u=-0.5 U(x-a)$. Similarly when a pair of forces of magnitude 0.5 is applied at $x=-2 a$ and $x=0$ the displacement field is $u=0.5 U(x+a)$. By superposing these two solutions and letting $a \rightarrow \infty$, we find that the displacement field corresponding to a single concentrated force of unit magnitude applied at $x=0$ is 


$$
\widehat{U}(x)=\frac{1}{2} \lim _{a \rightarrow \infty}\{U(x+a)-U(x-a)\} .
$$

From (40), the function defined by (45) solves the single concentrated load problem, i.e., the solution corresponding to

$$
b(x)=\delta(x) .
$$

Evaluating the displacement field (45) using (43) leads to

$$
\widehat{U}(x)=\widehat{U}_{0}(x)+\frac{\mathrm{i} \ell}{6 E} \sum_{j= \pm 1, \pm 2, \ldots} \frac{z_{j} \mathrm{e}^{\mathrm{i} z_{j}|x| / \ell}}{1-\cos z_{j}}+\frac{\delta(x)}{P},
$$

where

$$
\widehat{U}_{0}(x)=-\frac{|x|}{2 E} .
$$

Equation (47) provides a Green's function that can be used for constructing a solution to the general load problem. Using (47), the displacement field corresponding to an arbitrary body force distribution $b(x)$ is

$$
u(x)=\int_{-\infty}^{\infty} b\left(x^{\prime}\right) \widehat{U}\left(x-x^{\prime}\right) \mathrm{d} x^{\prime} .
$$

This provides an alternative approach to the Fourier transform method discussed in Section 2.

The limit of vanishing length scale $\ell \rightarrow 0$, leads to the classical result $u=$ $\widehat{U}_{0}(x)$. It is worth noting that in the classical theory of elasticity, the displacement field associated with this problem is bounded and continuous at all points in the bar. In contrast, as seen from (47), the linear peridynamic theory predicts unbounded displacements at the point where the force is applied.* This is true for the peridynamic theory in three dimensions as well. It is perhaps worth recalling that the solution to the three-dimensional problem of a concentrated load in the classical theory of elasticity (Kelvin's problem) involves unbounded displacements at the point of force application [5].

\section{Propagation of Discontinuities}

In general, the equilibrium solution for the displacement field $u$ has the same smoothness as the body force field $b$. This follows immediately from (23). Note that this represents a difference between the peridynamic theory and the classical theory of elasticity, where in the latter, $u$ would be two orders smoother than $b$.

In addition, any discontinuity in the micromodulus $C$ (or in one of its derivatives), has a further effect on the smoothness of $u$ as we now demonstrate. Suppose

\footnotetext{
$\star$ This is a consequence of the fact that in the peridynamic theory, in general, the displacement field and body force field typically have the same smoothness according to (23).
} 
that $b$ has a discontinuity in its $N$ th derivative at some $x=x_{b}$ (which because of (23) means that $u$ will necessarily have a discontinuity in its $N$ th derivative at $x=x_{b}$ ), and that in addition, $C$ has a discontinuity in its $L$ th derivative at some $x=x_{c}$. Then we will show that $u$ has a discontinuity in its $(N+L+1)$ th derivative at $x=x_{b}+x_{c}$; in its $(N+2 L+2)$ th derivative at $x=x_{b}+2 x_{c}$, and so on, so that in general, it has a discontinuity in its $(N+n L+n)$ th derivative at $x=x_{b}+n x_{c}$, $n=1,2, \ldots$. The smoothness of $u$ therefore increases as one moves away from the loading region. In general, it is the order $L$ of the discontinuous derivative of $C$ that determines the rate at which the smoothness of $u$ increases.

In order to demonstrate this, let the jump in a function $p$ at a location $x$ be denoted by

$$
[p]_{x}=\lim _{\epsilon \rightarrow 0}(p(x+\epsilon)-p(x-\epsilon)) .
$$

It is convenient to write the equation of equilibrium (23) in the form

$$
P u(x)=\int_{-\infty}^{\infty} C(\xi) u(x-\xi) \mathrm{d} \xi+b(x) \quad \forall x,
$$

where we have set $P=\int_{-\infty}^{\infty} C(\xi) \mathrm{d} \xi$; in fact $P=\bar{C}(0)$ and we assume this to be nonzero. Taking the $R$ th derivative of (51) with respect to $x$ gives

$$
P u^{(R)}(x)=\int_{-\infty}^{\infty} C(\xi) u^{(R)}(x-\xi) \mathrm{d} \xi+b^{(R)}(x),
$$

where, for any function $p$, we adopt the notation

$$
p^{(0)}(x)=p(x), \quad p^{(n)}(x)=\int^{x} p^{(n+1)}\left(x^{\prime}\right) \mathrm{d} x^{\prime}, \quad n=0, \pm 1, \pm 2, \ldots,
$$

so that $p^{(-1)}=\int^{x} p\left(x^{\prime}\right) \mathrm{d} x^{\prime}, p^{(1)}=\mathrm{d} p / \mathrm{d} x$ and so on. Integrating (52) by parts $L$ times gives

$$
P u^{(R)}(x)=\int_{-\infty}^{\infty} C^{(L)}(\xi) u^{(R-L)}(x-\xi) \mathrm{d} \xi+b^{(R)}(x) .
$$

At this point it is helpful to change the dummy integration variable to $x^{\prime}=x-\xi$ :

$$
P u^{(R)}(x)=\int_{-\infty}^{\infty} C^{(L)}\left(x-x^{\prime}\right) u^{(R-L)}\left(x^{\prime}\right) \mathrm{d} x^{\prime}+b^{(R)}(x) .
$$

We can evaluate the jump of (55) at any $x$ by using (50). This leads to

$$
P\left[u^{(R)}\right]_{x}=\int_{-\infty}^{\infty}\left[C^{(L)}\right]_{x-x^{\prime}} u^{(R-L)}\left(x^{\prime}\right) \mathrm{d} x^{\prime}+\left[b^{(R)}\right]_{x} .
$$

Suppose that $C^{(L)}$ contains a finite number of jumps. Now suppose that $u^{(R-L-1)}$ is discontinuous at exactly one point $x^{*}$. Then its derivative contains a delta function centered at $x^{*}$ and so we can write

$$
u^{(R-L)}(x)=\left[u^{(R-L-1)}\right]_{x^{*}} \delta\left(x-x^{*}\right)+\phi(x),
$$


where $\phi$ is some continuous function. On substituting (57) into (56), and evaluating the integral using the properties of the delta function, one obtains

$$
P\left[u^{(R)}\right]_{x}=\left[C^{(L)}\right]_{x-x^{*}}\left[u^{(R-L-1)}\right]_{x^{*}}+\left[b^{(R)}\right]_{x} .
$$

To put this in a slightly more suggestive form, set $N=R-L-1$. Then (58) may be rewritten as

$$
P\left[u^{(N+L+1)}\right]_{x}=\left[C^{(L)}\right]_{x-x^{*}}\left[u^{(N)}\right]_{x^{*}}+\left[b^{(N+L+1)}\right]_{x}
$$

which holds for all $x$. This means that a jump in some derivative of $u$ can occur at a location $x$ for either of two reasons. First, there might be a jump in that same derivative of $b$ at that same location. Second, there might be a jump in a derivative of $u$ at some nearby point $x^{*}=x-x_{c}$ provided there is also a jump in some derivative of $C$ at $x_{c}$.

To be more specific, suppose that $u^{(N)}$ is discontinuous at $x=x_{b}$ because $b$ has a corresponding discontinuity at this location. Suppose further that $C^{(L)}$ is discontinuous at $x=x_{c}$. Then from (59), setting $x^{*}=x_{b}$ and $x=x_{b}+x_{c}$, shows that $u^{(N+L+1)}$ is discontinuous at this $x$ (even though $b$ might not have any discontinuity at this location). Applying (59) again with $N$ replaced by $N+L+1$ and this time setting $x^{*}=x_{b}+x_{c}$ and $x=x_{b}+2 x_{c}$ shows that $u^{(N+2 L+2)}$ has a discontinuity at this $x$. One can continue this process indefinitely showing that, in general, $u^{(N+n L+n)}$ has a discontinuity at $x_{b}+n x_{c}$ for $n=1,2, \ldots$ The discontinuity propagates in this way, but the discontinuous derivatives have higher and higher order as one moves away from the loading region. In this sense, the discontinuities weaken as their distance from the loading region increases. This propagation is illustrated schematically in Figure 3.

As an example of this process, we return to the example of two concentrated forces discussed in Section 4. In this case the locations of the discontinuities are $x_{b}=a$ and $x_{c}=\ell$. From (38), it follows that $\left[C^{(0)}\right]_{\ell}=-3 E \ell^{-3}$. Since the body force consists of delta functions at $x= \pm a$, it immediately follows that $\left[b^{(-1)}\right]_{ \pm a}=1$. Applying (59) with $L=0, N=-2$, and $x=x^{*}=a$ leads to

$$
\left[U^{(-1)}\right]_{a}=\frac{1}{P}\left[b^{(-1)}\right]_{a}=\frac{\ell^{2}}{6 E},
$$

where $P=\bar{C}(0)=6 E / \ell^{2}$ has been obtained from (39). Using this result and reapplying (59) with $N=-1, x=a+\ell, x^{*}=a$ leads to

$$
\left[U^{(0)}\right]_{a+\ell}=\frac{1}{P}\left[C^{(0)}\right]_{\ell}\left[U^{(-1)}\right]_{a}=-\frac{\ell}{12 E} .
$$

Repeating the process with $N=0, x=a+2 \ell, x^{*}=a+\ell$ results in

$$
\left[U^{(1)}\right]_{a+2 \ell}=\frac{1}{P}\left[C^{(0)}\right]_{\ell}\left[U^{(0)}\right]_{a+\ell}=\frac{1}{24 E}
$$




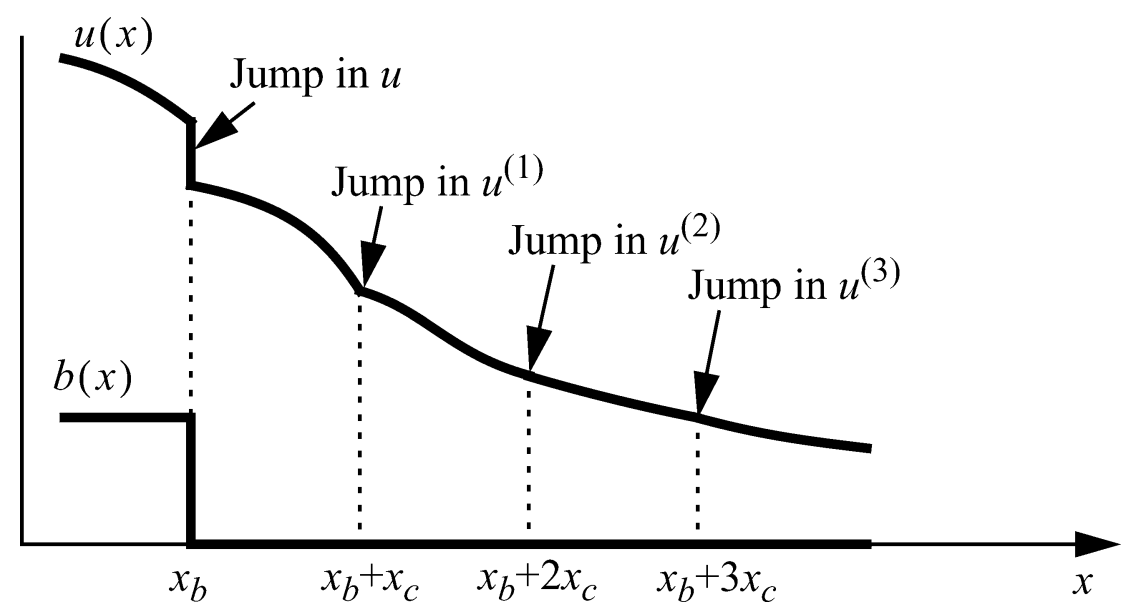

(a)

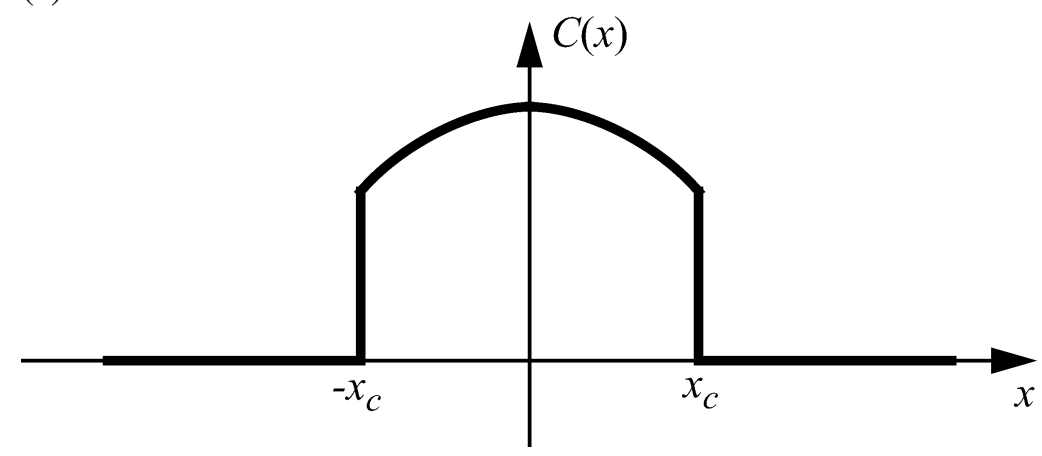

(b)

Figure 3. Decay of discontinuities in displacement to the right of the loading region in response to step function loading (a). $C$ contains discontinuities as shown (b).

and in general

$$
\left[U^{(n)}\right]_{a+(n+1) \ell}=\frac{\ell^{2}}{6 E}\left(\frac{-1}{2 \ell}\right)^{n+1}, \quad n=-1,0,1,2, \ldots
$$

This demonstrates the propagation of discontinuities in the derivatives of $U$ of increasing order moving away from the points of application of the point load.

In the classical theory of elastostatics, the displacement field can have discontinuities in its first derivative only if the elasticity tensor is such that ellipticity of the partial differential equations can be lost, which is closely related to the condition of nonconvexity of the elastic potential. ${ }^{\star}$ In one dimension, these conditions generally require nonmonotonicity of the stress-strain curve of a bar. In the classical theory,

\footnotetext{
* Conditions for and consequences of these conditions have been studied by Ericksen [6], Ball [7], Knowles and Sternberg [8], Abeyaratne [9], Kikuchi and Triantafyllidis [10], Rosakis [11], and Rosakis and Jiang [12] among others.
} 
if the stress-strain relation in an elastic material is monotonic, it follows from the equilibrium equation that the displacement field is two derivatives smoother than the body force field. In contrast, in the peridynamic theory the displacement field and body force field have the same smoothness. Consequently, as the results of this section show, a loss of ellipticity is not required for discontinuities to exist in the peridynamic theory, since discontinuities can be stimulated by discontinuities in the body force density and propagated by discontinuities in the micromodulus function $C$.

It was shown previously in Section 3 that oscillations due to loading decay as one moves away from the loading region. The results of the present section show that in a qualitatively similar fashion, the displacement field also becomes smoother far from the loading region.

\section{Discussion}

The results described above show that the peridynamic theory predicts some features in an apparently simple one-dimensional equilibrium problem that would not be present in the classical solution. Among these are a pattern of wavelike oscillations that spread out to infinity from the loading region. These oscillations decay far from the loading region, provided the material properties are reasonable, i.e., the wave speeds are positive. Another interesting feature is a repeating pattern of discontinuities in higher and higher derivatives as one moves away from the loading region. The way these discontinuities propagate depends on the material properties through the micromodulus function $C$. There is no analogue to this repetition of discontinuities in the classical theory, at least not for elliptic materials.

The decay in the amplitude of the oscillations and the order of the discontinuities they contain away from the loading region amounts to a kind of St. Venant principle in one dimension for peridynamic materials with positive wave speeds. This version of the St. Venant principle is comprised of the decay rates for oscillations and discontinuities derived in (37) and (59) away from the loading region.

Finally, it is reasonable to ask what exactly is to be gained by using the peridynamic model in the one-dimensional equilibrium bar problem that has such a simple, and to all appearances adequate, solution in the classical theory. The peridynamic model has two potential advantages: First, it allows for the spontaneous emergence of discontinuities, in contrast to the classical theory, which predicts deformations with infinite smoothness in typical (elliptic) materials. Second, it includes long-range forces between material particles, unlike the classical theory, which generally deals only with contact forces between particles.

When viewed as a continuum version of molecular dynamics, the peridynamic theory may be capable of modeling events on a small scale that go unseen by the classical theory of elasticity. This is particularly true of those microscale features that involve the spontaneous emergence of discontinuities such as cracks and other sources of material failure. For example, consider two identical bars of length $H$ 
that are brought into contact with each other at the ends, and then subjected to compressive loads at the free ends. The classical theory would predict a homogeneous deformation as though there were a single bar of length $2 H$, thus ignoring the point of contact. In contrast, a peridynamic model of this problem could take into account the alteration in the interparticle forces in the vicinity of the contact region. Presumably, the short-range forces on opposite sides of the discontinuity would be increased at the expense of the long-range forces. This local perturbation in the forces could be modeled, as a first approximation, as a self-equilibrated load problem with loads exerted on each bar in the vicinity of the contact surface. This loading would be reflected in the patterns of oscillations and discontinuities similar to those described in this paper.

If $H$ is on the order of 1 meter, the adequacy of the classical model is perhaps self-evident. On the other hand, if $H=10^{-8} \mathrm{~m}$, which is not an uncommon length scale in many practical problems of current interest, it is less obvious that the classical solution provides a good representation. At this length scale, it is likely that long-range forces of the type in the peridynamic approach would need to be considered in an accurate continuum model.

It has been observed experimentally that the effective Young's modulus and strength of nanoscale fibers and tubes are greater than the corresponding macroscale values [14]. Multiple physical effects, not all of them fully understood, contribute to this excess stiffness and strength. There is currently interest in the mechanics community in developing continuum models for these small-scale structures. As discussed Section 1, the peridynamic theory explicitly includes long-range forces, and its specialization to extension of a bar takes into account the cross-sectional geometry through (4). It therefore seems plausible that the peridynamic theory, when used with a suitable atomistic force density function $\mathbf{f}$, could offer advantages for physically based continuum modeling of these nanoscale structures.

As noted in the introduction, one of the most important characteristics of the peridynamic theory of elasticity is the fact that it does not involve spatial derivatives of the displacement field. It is worth noting however that in the special case of a smooth motion where one can, for example, define strain, there is a formal relation between this theory and a nonlocal theory of elasticity. In order to see this, consider a homogeneous, microelastic body, and let $K(\xi)$ be a constitutive function defined through

$$
K^{\prime \prime}(\xi)=C(\xi) .
$$

Substituting (64) into the equation of motion (11), integrating once by parts, and setting $\varepsilon=\partial u / \partial x$ leads to

$$
\frac{\mathrm{d}}{\mathrm{d} x} \int_{-\infty}^{\infty} K\left(x^{\prime}-x\right) \varepsilon\left(x^{\prime}, t\right) \mathrm{d} x^{\prime}+b(x, t)=\rho \ddot{u}(x, t)
$$

provided that $K^{\prime}(\xi)$ decays to zero sufficiently fast as $\xi \rightarrow \infty$. If we formally set

$$
\sigma(x, t)=\int_{-\infty}^{\infty} K\left(x^{\prime}-x\right) \varepsilon\left(x^{\prime}, t\right) \mathrm{d} x^{\prime},
$$


the peridynamic equation of motion (65) can now be written in the classical form $\partial \sigma / \partial x+b=\rho \ddot{u}$. Thus if we identify $\sigma$ with stress and $\varepsilon$ with strain, (66) corresponds to the constitutive relation for a certain class of nonlocal elastic materials with kernel $K$ (e.g., [13]). In the special case of a homogeneous deformation one can readily show by integrating by parts that (66) reduces to (19).

\section{Acknowledgements}

Sandia is a multiprogram laboratory operated by Sandia Corporation for the United States Department of Energy under Contract DE-AC04-94AL85000. This work was supported by the Joint DOE/DOD Munitions Technology Program, and by the U.S. Department of Energy. The authors thank Drs. Allen Robinson and Timothy Trucano of Sandia National Laboratories for reviewing the manuscript.

\section{References}

1. S.A. Silling, Reformulation of elasticity theory for discontinuities and long-range forces. J. Mech. Phys. Solids 48 (2000) 175-209.

2. R.J. Atkin and N. Fox, An Introduction to the Theory of Elasticity. (1980) Longman, London, pp. 186-187.

3. M.J. Lighthill, Fourier Analysis and Generalised Functions. Cambridge Univ. Press, Cambridge (1978).

4. E. Zauderer, Partial Differential Equations of Applied Mathematics. Wiley, New York (1983) pp. 365-376.

5. E. Sternberg, Three-dimensional stress concentrations in the theory of elasticity. Appl. Mech. Rev. 11(1) (1958).

6. J.L. Ericksen, Equilibrium of bars. J. Elasticity 5 (1975) 191-201.

7. J.M. Ball, Convexity conditions and existence theorem in nonlinear elasticity. Arch. Rational Mech. Anal. 63 (1977) 337-403.

8. J.K. Knowles and E. Sternberg, On the failure of ellipticity and the emergence of discontinuous deformation gradients in plane finite elastostatics. J. Elasticity 8 (1978) 329-379.

9. R.C. Abeyaratne, Discontinuous deformation gradients in plane finite elastostatics of incompressible materials. J. Elasticity 10 (1980) 255-293.

10. N. Kikuchi and N. Triantafyllidis, On a certain class of elastic materials with non-elliptic energy densities. Quart. Appl. Math. 40 (1982) 241-248.

11. P. Rosakis, Ellipticity and deformations with discontinuous gradients in finite elastostatics. Arch. Rational Mech. Anal. 109 (1990) 1-37.

12. P. Rosakis and A. Jiang, Deformations with discontinuous gradients in plane elastostatics of compressible solids. J. Elasticity 33 (1993) 233-257.

13. A.C. Eringen, Vistas of nonlocal continuum physics. Internat. J. Engrg. Sci. 40 (1992) 15511565.

14. M.M.J. Treacy, T.W. Ebbesen and J.M. Gibson, Exceptionally high Young's modulus observed for individual carbon nanotubes. Nature 381 (1996) 678-680. 